\title{
Adjuvante Therapie mit einer N-Palmitoylethanol- amin-haltigen Creme bei Kindern mit atopischem Ekzem (ATOPA-Studie) ${ }^{*}$
}

\section{Adjuvant Treatment of Children with Atopic Eczema with an Emollient Containing N-Palmitoylethanolamine (ATOPA Study)}

\author{
Autoren \\ B. Eberlein ${ }^{1}$, C. Eicke ${ }^{2}$, H.-W. Reinhardt ${ }^{3}$, J. Ring \\ Institute \\ Klinik und Poliklinik für Dermatologie und Allergologie am Biederstein, Technische Universität München \\ Bad Vilbel \\ Stiefel Laboratorium GmbH, Offenbach
}

Bibliografie

DOI 10.1055/s-0028-1103406

Online-Publikation: 27.11.2008

Akt Dermatol 2009; 35:

339-341 @ Georg Thieme

Verlag KG Stuttgart · New York ISSN 0340-2541

Korrespondenzadresse

Prof. Dr. Bernadette Eberlein

Klinik und Poliklinik für

Dermatologie und Allergologie

am Biederstein

Biedersteiner Str. 29

80802 München

eberlein@Irz.tum.de

\section{Zusammenfassung}

$\nabla$

Ziel dieser Studie war es, Daten zu hautpflegenden Eigenschaften und zu Parametern der Lebensqualität bei Patienten mit atopischem Ekzem zu erhalten während der Anwendung einer N-Palmitoylethanolamin-haltigen Creme mit einer einzigartigen lamellären Matrix. In diese multizentrische nicht-kontrollierte prospektive Kohortenstudie wurden 2456 Patienten (923 Kinder $\leq 12$ Jahre) eingeschlossen. Die Beobachtungsdauer betrug 38 Tage.

Die kombinierte Auswertung von Erythem, Pruritus, Exkoriation, Schuppung, Lichenifikation und Trockenheit ergab eine signifikante Verbesserung der Symptome um 58,6\% (57,7\% bei Erwachsenen $>12$ Jahre und $60,5 \%$ bei Kindern $\leq 12$ Jahre). Unter Verwendung einer visuellen Analog-Skala berichteten die Patienten von einer signifikanten Linderung des Juckreizes bereits 6 Tage nach Stu-

\section{Einleitung}

$\nabla$

Das atopische Ekzem gehört zu den häufigsten Hauterkrankungen. Die Prävalenz liegt zwischen $2 \%$ und 5\% bei Erwachsenen und bei etwa 15\% bei Kindern und Jugendlichen [1,2]. Zur Basistherapie gehört die Langzeitanwendung geeigneter Hautpflegecremes. Ein innovativer Ansatz zur Hautpflege ist die Verwendung einer Kortikosteroid-freien Barriere-Reparatur-Creme, die auf Grundlage einer lamellaren Matrix (DermaMembran-Struktur, DMS ${ }^{\circledR}$ ) aufbaut und gleichzeitig N-Palmitoylethanolamin (PEA) als antientzündliche Komponente enthält. Diese Creme enthält keine irritierenden Substanzen wie Emulgatoren, Konservierungsstoffe, Farbstoffe oder Parfüm. Ziel der ATOPA-Studie war es, die Wirk${ }^{*}$ Gehalten als Vortrag beim 2. Wiesbadener Symposium
Kinderdermatologie, 12.11.2008. dienbeginn und von einer weiteren Abnahme zu Studienende. Die Schlafqualität besserte sich ebenfalls signifikant im Studienverlauf. Topische Kortikosteroide wurden bei $56 \%$ aller Patienten (53,4\% bei Erwachsenen $>12$ Jahre und $62,5 \%$ bei Kindern $\leq 12$ Jahre) abgesetzt. Die wöchentliche Applikationsrate kortisonhaltiger Cremes sank im Studienverlauf von 7,9 auf 3,0 Anwendungen. Die Verträglichkeit wurde von Ärzten wie Patienten in $92 \%$ der Fälle als sehr gut oder gut eingestuft.

Zusammenfassend zeigt diese Studie eine relevante Verbesserung objektiver und subjektiver Parameter des atopischen Ekzems unter regelmäßiger Anwendung der Studiencreme über einen Zeitraum von 38 Tagen. Der Bedarf topischer Kortikosteriode wurde erheblich gesenkt, wodurch sich auch wichtige pharmakoökonomische Vorteile für das Management des atopischen Ekzems ergeben können.

samkeit dieser Creme bei Neurodermitis zu dokumentieren.

\section{Methoden}

$\nabla$

Es handelte sich um eine multizentrische, nichtkontrollierte, prospektive Kohortenstudie, die in Brasilien, Deutschland, den Philippinen und Spanien zwischen November 2004 und April 2005 durchgeführt wurde. Einschlusskritierien waren trockene und juckende Haut seit mindestens 12 Monaten sowie ein sichtbares Ekzem gemäß den Kriterien der United Kingdom Working Party [3]. Ausschlusskritierien waren ein schwerer oder akuter Schub des atopischen Ekzems, Nässen und Krustenbildung. Die Patienten wurden mit der Studiencreme (Physiogel A.I. ${ }^{\circledR}$ Creme) für den Studienzeitraum (4-6 Wochen) versorgt. 


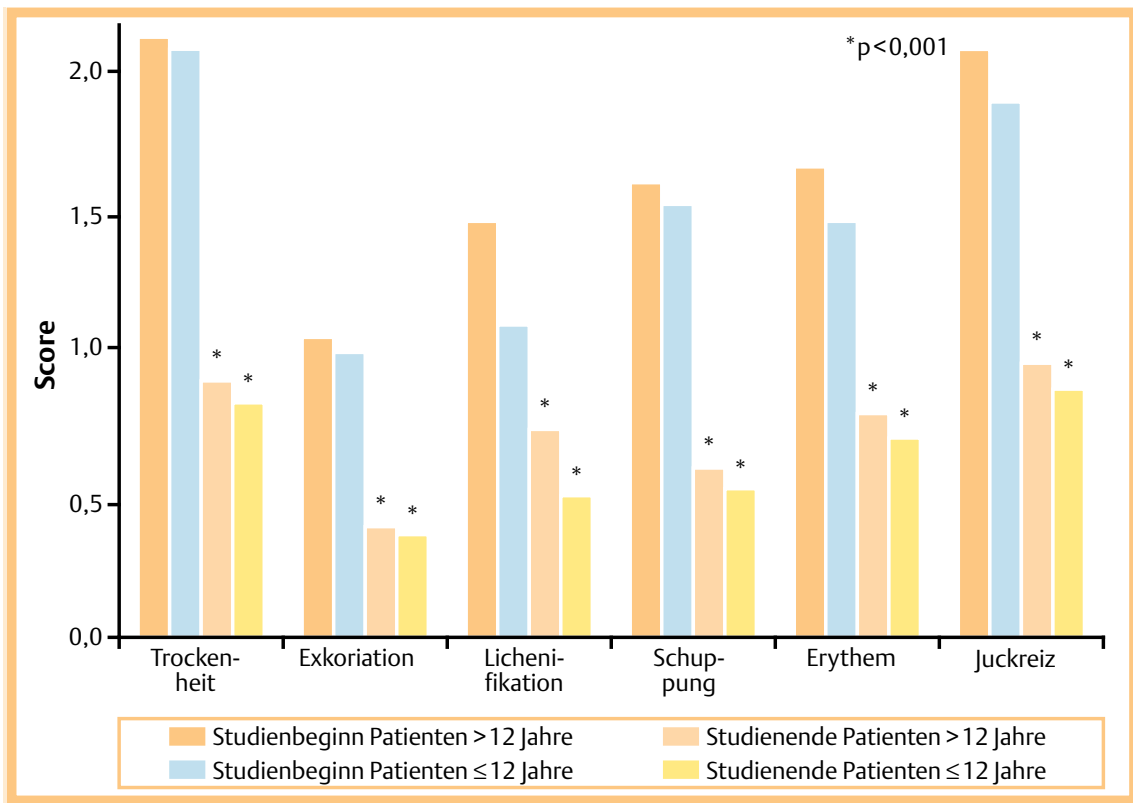

Abb. 1 Klinische Symptome nach Einschätzung durch die Ärzte $\left({ }^{*} p<0,001\right)$.
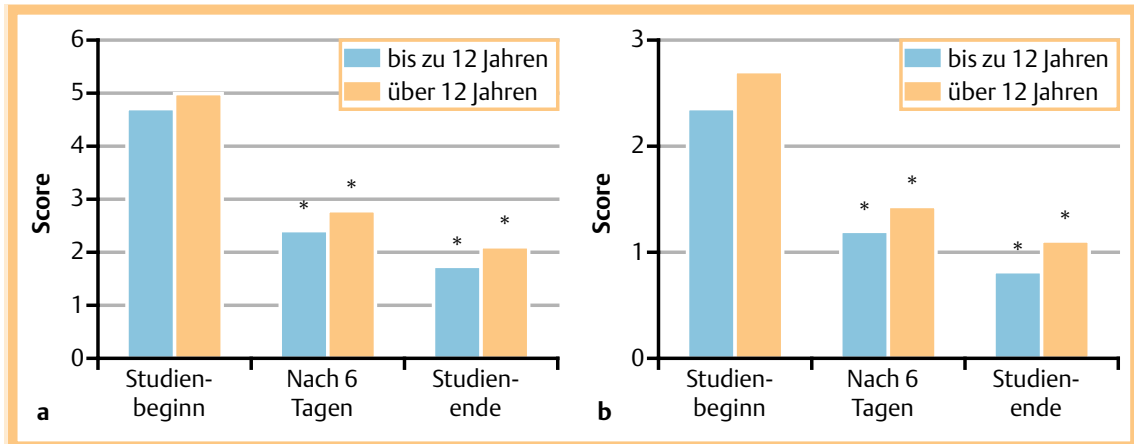

Abb. 2 a Juckreiz gemäß der Bewertung durch die Patienten auf einer visuellen Analogskala b Schlafverlust gemäß der Bewertung durch die Patienten auf einer visuellen Analogskala ( ${ }^{*} p<0,001$ versus Ausgangswert).

Der Schweregrad des Ekzems wurde vom Arzt durch Bewertung von Trockenheit, Exkoriation, Lichenifikation, Schuppung, Erythem, und Pruritus anhand einer 4-Punkte Skala (von $0=$ nicht vorhanden bis 3 = schwer) beurteilt. Diese Untersuchungen wurden zu Studienstart und -ende vorgenommen.

Die Intensitäten von Juckreiz und Schlaflosigkeit wurden anhand einer visuellen Analogskala von den Patienten selbst bewertet. Diese Bewertungen erfolgten zu Studienstart, nach 6 Tagen und zu Studienende mithilfe von Patientenfragebögen unabhängig vom Arzt. Zu Studienstart und -ende gaben die Patienten außerdem die Häufigkeit an, wie oft in den vergangenen 7 Tagen Kortison-haltige Cremes angewendet wurden.

\section{Ergebnisse \\ $\nabla$}

Es wurden die Daten von 2456 Patienten aus 525 Zentren (Pädiater und Dermatologen) ausgewertet, wobei das Alter des Gesamtkollektivs von 2 bis 70 Jahren reichte. Im Studienkollektiv befanden sich 923 Kinder bis zu 12 Jahren mit einem Durchschnittsalter von 5,5 Jahren. Die Rücklaufraten für die zwei Patientenfragebögen betrugen 96,3\% für den Studienstart/Tag 6 und 89,5\% für das Studienende. Die durchschnittliche Beobachtungsdauer lag zwischen 38 Tagen gemäß Arztangaben und 39 Tagen gemäß Patientendaten.

Die ärztlichen Untersuchungen ergaben signifikante Abnahmen der Schweregrade von Trockenheit, Exkoriation, Lichenifikation,
Schuppung, Erythem und Pruritus sowohl für Patienten über 12 Jahre als auch für die jüngeren Patienten ( $\bullet$ Abb. 1).

Der kombinierte Symptomen-Score fiel im Gesamtkollektiv von 9,68 auf 4,01 ab, was einer Verbesserung um 58,6\% entspricht $(\mathrm{p}<0,001)$. Von 192 Kindern, die zu Studienbeginn topische Steroide benutzten, konnten 120 (62,5\%) die Behandlung während der Studie absetzen. Von 367 Kindern, die keine Steroide zu Studienbeginn benutzten, begannen 28 Patienten (7,6\%) mit der Steroidbehandlung während der Studie.

Die Patienten berichteten von Abnahmen um 60\% sowohl der Pruritus-Intensität wie dem Ausmaß der Schlaflosigkeit ( $\bullet$ Abb. 2 a u. b).

Eine deutliche Besserung der Beschwerden gaben 47\% der Patienten unter 12 Jahren an, bei Patienten über 12 Jahren waren es 35\%. Von 410 Patienten aus dem Gesamtkollektiv, die zu Studienbeginn topische Steroide benutzten, konnten 219 (53,4\%) die Behandlung während der Studie absetzen. Patienten, die keine Steroide zu Studienbeginn benutzten, begannen in $11 \% \mathrm{mit}$ der Steroidbehandlung während der Studie. Von 192 Kindern, die zu Studienbeginn topische Steroide benutzten, konnten 120 $(62,5 \%)$ die Behandlung während der Studie absetzen. Von 367 Kindern, die keine Steroide zu Studienbeginn benutzten, begannen 28 Patienten (7,6\%) mit der Steroidbehandlung während der Studie.

In der Summe traten 235 unerwünschte Ereignisse bei $187 \mathrm{~Pa}-$ tienten (7,6\%) auf. Die Studienärzte bewerteten den Zusammenhang mit dem Studienprodukt als sicher oder wahrscheinlich in 
2,3\% aller Patienten. In allen diesen Fällen war die Haut betroffen, mit Brennen, Rötungen und Jucken als häufigste Symptome. Es wurde von keinem schwerwiegenden unerwünschten Ereignis berichtet.

Die Verträglichkeit wurde von Patienten und Ärzten in $92 \%$ der Fälle als sehr gut bis gut bewertet.

\section{Schlussfolgerung \\ $\nabla$}

Diese Studie zeigt, dass sich objektive und subjektive Symptome des atopischen Ekzems nach 38-tägiger Anwendung von Physiogel A.I. erheblich verbesserten. Schlaflosigkeit und Juckreiz, beides Marker für die Lebensqualität, verbesserten sich signifikant. Ebenso reduzierte sich der Bedarf an topischen Kortikosteroiden signifikant. Kinder zeigten etwas bessere Ansprechraten als Erwachsene. Die offensichtlichen anti-irritativen Eigenschaften, wie sie von Ärzten wie Patienten beobachtet wurden, sind möglicherweise einerseits auf die Barriere-stärkende lamellare Struktur der Studiencreme und andererseits auf die antipruriginösen und anti-inflammatorischen Eigenschaften des Inhaltsstoffs N-Palmitoylethanolamin [4] zurückzuführen. Weitere Studien sind nötig, um die spezifische Wirkung dieser Creme im Unterschied zu anderen Hautpflegecremes herauszustellen.

\section{Abstract}

\section{Adjuvant Treatment of Children with Atopic Eczema with an Emollient Containing N-Palmitoylethanol- amine (ATOPA Study) $\nabla$}

The objective of this study was to yield data on the skin care properties of a cream with a unique lamellar matrix containing $\mathrm{N}$-palmitoylethanolamine, and to assess quality of life variables during the management of patients with atopic eczema. In this multi-national, multicenter, observational, non-controlled, prospective cohort study 2456 patients (923 children $\leq 12$ years) were enrolled. Outcome was followed over 38 days of adjuvant treatment with the study cream.

Combined analysis of erythema, pruritus, excoriation, scaling, lichenification and dryness showed a significant score reduction by $58.6 \%$ at study end for all patients ( $57.7 \%$ for adults $>12$ years and $60.5 \%$ for children $\leq 12$ years). Patients reported a significant reduction of pruritus on visual analogue scales as soon as 6 days after starting treatment and a further reduction at study end. Sleep quality improved significantly during the study period. Earlier used topical corticosteroids were omitted by $56 \%$ of all patients ( $53.4 \%$ in adults $>12$ and $62.5 \%$ in children $\leq 12$ years) at study end and the average weekly application rate decreased from 7.9 to 3. The tolerance was assessed as very good or good in $92 \%$ of cases by both patients and doctors.

This study demonstrates substantial relief of objective and subjective symptoms of atopic eczema after regular skin care with the study cream over a period of 38 days. The requirement for treatment with topical corticosteroids was reduced in a substantial proportion of participants, which has potentially important pharmacoeconomic implications in the management of atopic eczema.

Literatur

1 Ring J, Przybilla B, Ruzicka T(eds). Handbook of Atopic Eczema. Heidelberg: Springer, 2005

2 Ring J. Allergy in Practice. Heidelberg: Springer, 2004

3 Williams HC. On the definition and epidemiology of atopic dermatitis. Dermatol Clin 1995; 13: 649-657

4 Ständer S, Reinhardt HW, Luger TA. Topische Cannabinoidagonisten. Eine effektive, neue Möglichkeit zur Behandlung von chronischem Pruritus. Hautarzt 2006; 57: $801-807$ 